\title{
Les Parasites des Chiroptères
}

\author{
Rôle épidémiologique \\ chez les Animaux et l'Homme au Katanga (1) \\ par Michel ANCIAUX de FAVEAUX (2)
}

Le but de cette étude est de passer en revue les infections et les maladies provoquées, directement ou indirectement, par les Chiroptères et leurs parasites.

Nous examinerons surtout les résultats obtenus dans une région bien déterminée: le Haut-Katanga (République du Congo).

\section{I. - OBSERVATIONS ECOLOGIQUES SUR LES CHIROPTERES}

Nos recherches dans le Haut-Katanga (carte 1), et plus particulièrement dans les environs de Jadotville (carte 2), ont porté sur les biotopes suivants : domaine souterrain (grottes, galeries artificielles de prospection minière, canalisations), habitations (toits, cheminées, volets), arbres (troncs, feuilles) et nids d'Oiseaux.

\section{A. - Domaine souterrain.}

Ce milieu constitue notre terrain de prédilection (Anciaux de Faveaux 1950, 1956, 1959, 1960, 1964a). Depuis notre première étude d'ensemble sur les Chiroptères du Haut-Katanga (1958), nous avons élargi notre champ d'investigations: 11 réseaux hypogés comprenant 40 grottes et trois anciennes galeries minières. Une campagne biospéléologique, en août 1962, nous a permis d'explorer quelques autres cavités encore inédites.

1. Les Chiroptères cavernicoles forment le plus souvent des colonies très denses (3) :

a) PteropidÉs : Rousettus (Rousettus) aegyptiacus E. Geoffroy (1, 2, 5, 9 bis).

b) Emballonuridés: Taphozous sudani Thomas (2).

(1) Communication présentée au $\mathrm{XVI}^{e}$ Congrès international de Zoologie, à Washington, le 23 août 1963.

(2) B.P. 1121, Jadotville (République du Congo) cole.

(3) Les numéros inscrits sur les cartes sont repris dans notre exposé relatif à la faune caverni- 
c) RHINOLOPHIDÉs: Rhinolophus h. hildebrandti Peters (1, 2, 4, 5, 6, 7, 8, 9, 9 bis, 11) ; Rhinolophus landeri lobatus Peters (1, 2, 3, 4, 7, 9 bis); Rhinolophus simulator K. Andersen (1, 2, 4, 9, 9 bis, 12) ; Hipposideros caffer centralis K. Andersen (1, 2, $3,4,7,9,9$ bis, 12).

d) Vespertilionidés: Miniopterus inflatus Thomas (1, 2, 4, 5, 8, 9 bis, 10, 12) ; Miniopterus schreibersi natalensis A. Smith $(1,4,6,9,9$ bis, 11, 12).

2. Certaines espèces se composent d'individus isolés ou en petit nombre :

a) RHINOLOPHIDÉs : Rhinolophus blasii empusa K. Andersen (7); Rhinolophus clivosus zuluensis K. Andersen (2, 9 bis) ; Rhinolophus swynnii Gough (9 bis) ; Hipposideros commersoni gigas Wagner (1) ; Cloeotis percivali australis Roberts (4, 9, 12).

b) Vespertillonidés: Myotis tricolor Temminck (1, 4, 9 bis); Nycticeius (Scotoecus) hirundo hindei Thomas (2).

c) NyCteridés: Nycteris th. thebaica E. Geoffroy $(8,9$ bis).

3. D'autres fréquentent, en groupes plus ou moins importants, les souterrains artificiels: canalisations (S.A. 1: Keyberg-Elisabethville, route d'Elisabethville vers Kasenga ; S.A. 2 : Forge de la Mission catholique de Kapolowe; galeries minières de sondage (S.A. 3 : Likasi-Jadotville ; S.A. 4 : Kakanda ; S.A. 5 : Shinkolobwe) ; conduite forcée (S.A. 6: Mwadingusha).

a) RHINolophidÉs: Rhinolophus h. hildebrandti Peters (S.A. 4); Rhinolophus landeri lobatus Peters (S.A. 6) ; Hipposideros caffer centralis K. Andersen (S.A. 3).

b) VeSPERTILIONIDÉs: Miniopterus schreibersi natalensis A. Smith (S.A. 3).

c) NycteridÉs: Nycteris m. macrotis Dobson (S.A. 1, S.A. 5); Nycteris th. thebaica E. Geoffroy (S.A. 2).

4. Nous avons observé des espèces étroitement associées:

a) Rhinolophus landeri lobatus Peters et Hipposideros caffer centralis K. Andersen (3, 9 bis: grotte Kyasala).

b) Rhinolophus landeri lobatus Peters, Hipposideros caffer centralis $\mathrm{K}$. Andersen et Cloeotis percivali australis Roberts (4: grotte Dethioux).

c) Hipposideros caffer centralis K. Andersen et Rhinolophus blasii empusa K. Andersen (7).

d) Rhinolophus simulator K. Andersen et Nycteris th. thebaica E. Geoffroy (9 bis : grotte Lusolo).

e) Rhinolophus simulator K. Andersen, Hipposideros caffer centralis K. Andersen et Cloeotis percivali australis Roberts (12).

f) Miniopterus inflatus Thomas et Miniopterus schreibersi natalensis A. Smith (12).

g) Un Miniopterus schreibersi natalensis A. Smith ơ avec deux Myotis tricolor Temminck $\subsetneq$ au fond d'une crevasse pariétale (9 bis: grotte Kyamakonde). 


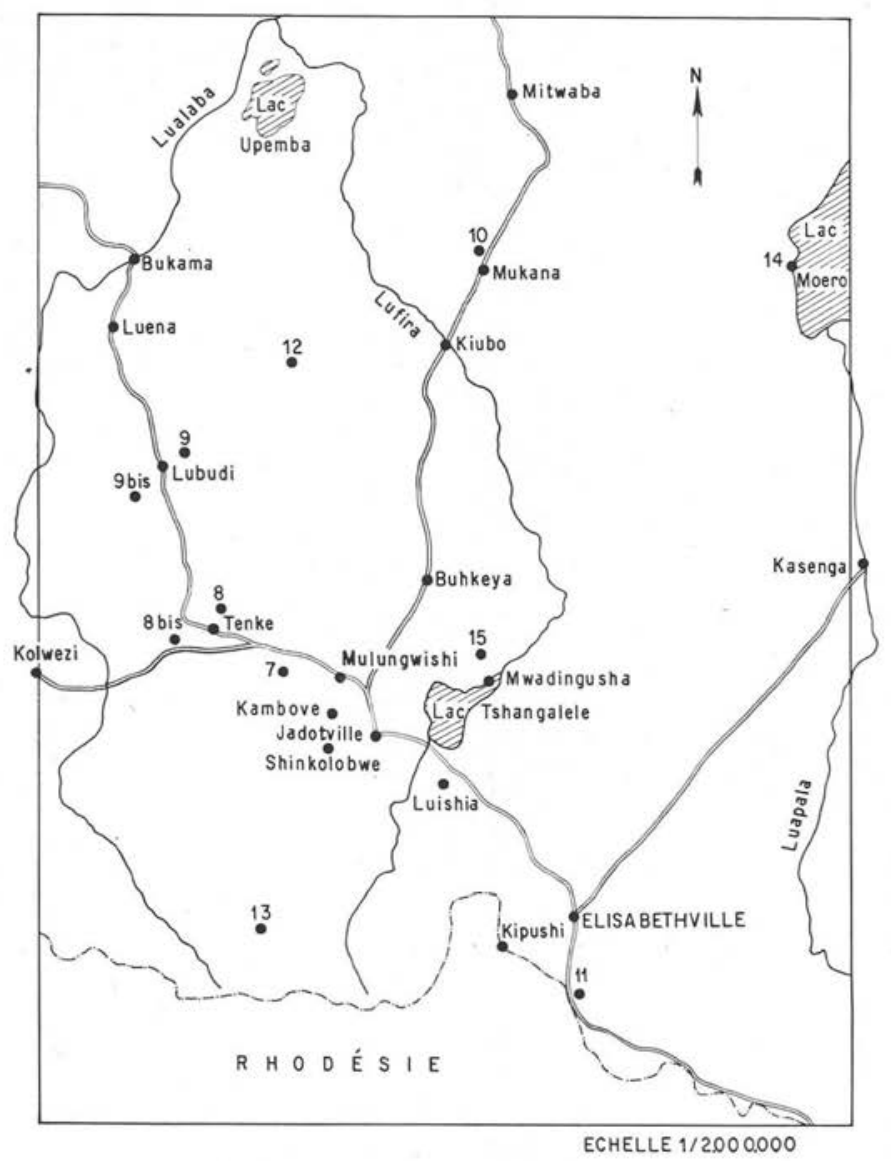

CARTE I (Régions explorées dans le Haut-Katanga)

7. Grotte de Kondo (près de Kakanda).

8. Grotte Tshamakele = Kamashinka : près de Mwela-Pande (Tenke). $8^{\text {bis }}$ Grottes dans le mont Kibwe: près de Kando (Tenke).

9. Grotte de Kaboyaboya: près de Kaio (Lubudi).

$9^{\text {bis }}$ Grottes près de Lubudi : - Lusolo, Kyasala, Mototo (à gauche de la route reliant Lubudi au barrage de Dikolongo). - Kyamakonde, Kando, Mpopola (près de Kwete-Lusambo). - Mulonga (à $23 \mathrm{kms}$ au S.W. de Lubudi). - Kyantapo (à $30 \mathrm{kms}$ au S.W. de Lubudi)

10. Grottes de Kiwakishi.

11. Grotte de Baya (à $30 \mathrm{kms}$ au Sud d'Elisabethville).

12. Grotte Kasoma (Parc National de l'Upemba) : rive droite de la rivière Kapandula.

13. Grotte Tshamalenge (aux sources du Lualaba).

14. Grotte de Kilwa (Lac Moero).

15. Grottes Mihogve et Kiantete (rivière Luivi, près de Mwandigusha). 


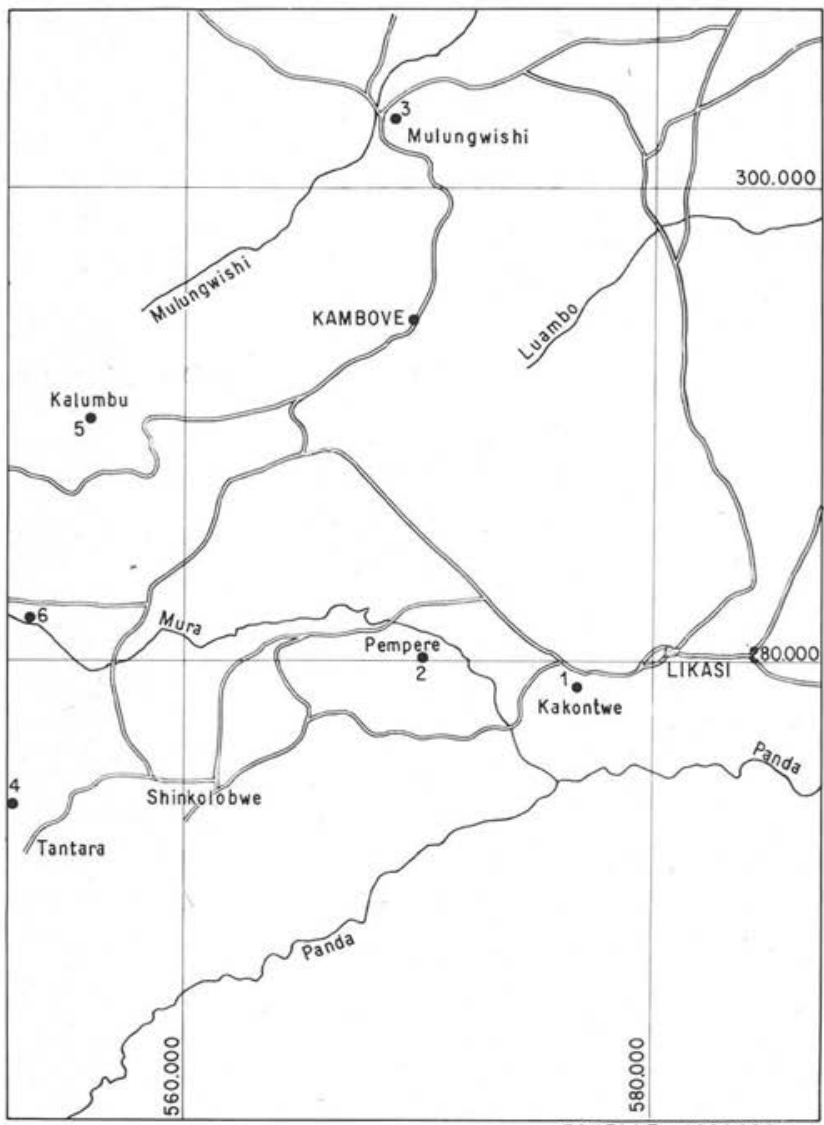

ECHELLE:1/200.000

CARTE II (Régions de Jadotville)

1. Grotte Kalomoni (près de Kakontwe): avec lac souterrain.

2. Grottes de Pempéré (au nombre de 13): Mwanga, Katembavikulu, Kasowena, etc... (à 15 kms de Jadotville)

3. Grottes de Mulungwishi (dans colline Kampemba).

4. Grottes de Tantara (près de Shinkolobwe) : - deux près de l'ancienne Ferme Dethioux. - deux au-dessus de la résurgence de la Kabonde (près du village Mwamba). - deux dans la vallée sèche de la Tantara

5. Grottes de Kalumbu (à $10 \mathrm{kms}$ de la mine de Kabolela).

6. Grottes dans le domaine Swanepoel (vallée de la Mura). 
B. - Habitations (toits, cheminées, volets).

a) PTÉRoPIDÉs: Epomophorus crypturus Peters (Kasapa, près d'Elisabethville); Epomophorus gambianus Ogilby (Kasenga).

b) Emballonuridés: Taphozous mauritianus E. Geoffroy (sous corniches de toits à Elisabethville et Kapolowe).

c) VeSPERTILIONIDÉS: Scotophilus n. nigrita Schreber (Elisabethville et Jadotville).

d) Molossidés (en colonies): Tadarida (Chaerephon) limbata Peters (Kapolowe, Kashiobwe). Tadarida (Mops) chitauensis Hill (Jadotville); Tadarida (Mops) condylura niveiventer Cabrera et Ruxton (Kapolowe, Kasenga, Kashiobwe, Keyberg, Elisabethville, Jadotville); Tadarida (Tadarida) ansorgei Thomas (usine électrique de Mwadingusha).

C. - Arbres (troncs, feuilles).

a) PTÉROPIDÉs : Epomophorus crypturus Peters (sur jacarandas à Elisabethville) ; Epomophorus gambianus Ogilby (Kakontwe, Kasenga, Jadotville); Epomops dobsoni Bocage (Elisabethville).

b) VeSPERTILIONIDÉs : Myotis (Selysius) bocagei Thomas, en étroite association avec Pipistrellus nanus Peters (dans feuilles en cornet de Bananiers, à Kakonde-Kapolowe) ; Pipistrellus nanus Peters (presque partout où il $\mathrm{y}$ a des Bananiers: dans les feuilles en cornet); Scotophilus n. nigrita Schreber (colonie dans un arbre creux à KipopoElisabethville); Laephotis wintoni angolensis Monard (sous écorce d'arbres à Musonge et Mumena : environs d'Elisabethville).

c) Molossidés : Tadarida (Chaerephon) spillmani Monard (sous écorce d'arbres morts, à Musonge et Kinke: environs d'Elisabethville).

D. - Nids d'Oiseaux.

VESPERTILIONIDÉs: Kerivoula a. argentata Tomes (dans un nid de républicains, à Kamatanda-Jadotville).

\section{II. - ENDOPARASITES}

\section{A) Bacilles.}

Pasteurella pestis a été signalé chez des Molossidés (Tadarida pumila Cretzochmar) €n Afrique Orientale et au Sénégal (Léger et Baury 1923).

Nous n'avons pas trouvé, Xenopsylla nubica Rothschild au Katanga ; cependant cette Puce a été récoltée à Pretoria (Afrique du Sud) sur Rhinolophus clivosus augur K. Andersen et sur Miniopterus sp. (De Meillon, Davis et Hardy 1961).

\section{B) Virus.}

1. Rage.

Recherches négatives en Nigérie chez Eidolon helvum Kerr (Boulger et Porterfield 1958 ; Boulger et Hardy 1960). 
Lors d'une épidémie de rage dans les régions d'Elisabethville et de Jadotville en 1958-1959, nous avons étudié le rôle possible des Chiroptères dans la propagation du virus avec la collaboration du Service vétérinaire du Congo à Elisabethville. 370 cerveaux et glandes salivaires de Chauves-souris (provenant surtout de la région de Jadotville) ont été inoculés intracérébralement à des souris blanches. Les espèces examinées ont été les suivantes: 46 Rousettus aegyptiacus E. Geoffroy, 2 Hipposideros caffer centralis K. Andersen, 10 Taphozous sudani Thomas, 23 Miniopterus inflatus Thomas, 150 Miniopterus schreibersi natalensis A. Smith, 3 Pipistrellus nanus Peters, 102 Tadarida (Mops) condylura niveiventer Cabrera et Ruxton, 30 Tadarida ansorgei Thomas, 4 Tadarida (Chaerephon) limbata Peters. L'absence de corps de Negri dans les préparations de cerveaux de souris après inoculation permet de conclure à l'inexistence du virus de la rage chez ces Chauves-souris.

Nous attirons l'attention, pour mémoire, sur une curieuse note de Constantine (1962) : cet auteur estime que l'air ambiant dans la « Frio Cave» (Texas) puisse jouer un rôle dans la transmission de la rage, là où vivent des Chiroptères en très grand nombre.

\section{Encéphalite.}

Lors de leurs recherches sur la rage en Nigérie, Boulger et Porterfield (1958) ont isolé un « arthropod-borne virus » (groupe B) : le «Lagos bat virus » chez Eidolon helvum Kerr.

Un virus Mengo a été détecté chez des Molossidés en Uganda (Collier 1955).

Rien au Katanga.

\section{FIÈVRE Q.}

Blanc et Bruneau (1957) ont découvert un virus de la fièvre Q, au Maroc, chez Eptesicus isabellinus Temminck. En Oubangui-Chari, en 1952, P. Giroud a été frappé de la fréquence des réactions pulmonaires fébriles chez des hommes vivant dans des paillottes abritant des chauves-souris; leur sérum agglutinait Rickettsia burneti à $1: 20$.

Les Tiques du genre Argas jouent un rôle appréciable dans les rickettsies (Suitor et Weiss, 1961). Argas (Chiropterargas) boueti Roubaud et Colas-Belcour est une espèce commune chez les Chiroptères d'Afrique (Roshdy 1962).

Rien au Katanga.

\section{Fièvre récurrente.}

Dans des grottes de Zanzibar, Garnham et Heisch (1953) ont rencontré des Spirochètes chez des Chiroptères. Novel (1954) cite comme réceptifs : Eidolon helvum Kerr, Epomophorus wahlbergi haldemani Halowell et Micropterus pusillus Peters (espèces signalées au Katanga).

Parmi les Tiques susceptibles de transmettre des Spirochètes en Afrique, notons : Ornithodoros graingeri Heisch et Guggisberg dans des grottes du Kenya, vecteur de Spirochaeta graingeri (Heisch 1953) ; Ornithodoros megnini Dugès au Katanga (Schoenaers 1950). 
Les chauves-souris pourraient assumer, dans l'épidémiologie des Spirochétoses humaines et animales, un rôle de réservoir de virus comparable à celui de quelques Insectivores et de nombreux Rongeurs.

C) Parasites sanguins.

\section{HAEMOSPORIDIES.}

\section{a) Plasmodium.}

Le seul vrai Plasmodium connu chez les Chiroptères est Plasmodium rousetti Van Riel et Hiernaux 1951, découvert dans le sang de Rousettus leachi A. Smith dans les grottes du Mont-Hoyo (Ituri : Congo). Nous ne l'avons pas observé chez les Roussettes du Katanga ; cependant un doute subsiste : un frottis de sang de Rousettus aegyptiacus E. Geoffroy présente des formes pigmentées assez voisines de l'espèce susdite.

\section{b) Hepatocystis.}

Des gamètes d'Hepatocystis epomophori Rodhain ont été trouvés par M. Lips et nous dans le sang d'Epomophorus crypturus Peters et d'Epomophorus gambianus Ogilby, à Elisabethville.

\section{c) Nycteria.}

Nycteria medusiformis Garnham et Heisch 1953 a été retrouvé par M. Lips et nous dans Nycteris macrotis Dobson (Lips et Rodhain 1956). Tout récemment, Garnham et al. (1963) ont observé un nouveau Nycteria : N. congolensis dans Rhinolophus hildebrandti Peters, au Kenya.

\section{d) Polychromophilus.}

Outre Polychromophilus melaniferus Dionisi dans le sang de Miniopterus inflatus Thomas (grottes de Kakontwe et Lubudi), nous avons découvert une nouvelle espèce inféodée aux Rhinolophidés (Rhinolophus hildebrandti Peters, Rhinolophus landeri lobatus Peters, Hipposideros caffer centralis K. Andersen) dans les grottes de Kakontwe et de Lubudi ; il s'agit de Polychromophilus congolensis Krampitz et Anciaux 1960.

Le 9 février 1957, nous avons injecté à une souris blanche du sang de Miniopterus inflatus Thomas (infecté par $P$. melaniferus Dionisi) ; trois jours après, la souris présentait des indices de fièvre et refusait de manger ; suite à une hémorragie anale, la souris est morte le 15 février 1957, et sa rate a montré de petits schizontes en formation.

\section{Flagellates: Trypanosomes.}

Trois espèces chez les Chiroptères du Katanga :

a) Trypanosoma leleupi Rodhain 1951: dans Hipposideros caffer centralis K. Andersen (grotte Kalomoni, à Kakontwe). Nous avons mis en évidence des Leptomonas et des formes crithidiennes chez Afrocimex leleupi Schouteden dans cette même grotte.

b) Trypanosoma pipistrelli, de type cruzi (Rodhain 1942): dans Miniopterus inflatus Thomas (grotte de Kakontwe). 
c) Trypanosoma thomasi Lips et Rodhain 1956: dans Nycteris macrotis Dobson (Keyberg-Elisabethville).

3. Microfilaires.

Des frottis de sang du poumon de Miniopterus inflatus Thomas (grotte Kyasala, à Lubudi) montrent des formes très nombreuses d'une espèce probablement inédite: jusqu'à huit œufs (avec embryons visibles) dans un même champ microscopique à diverses phases de développement ; les larves sont entourées d'une gaine très claire.

4. SPIROCHÈtes.

En plus de ceux qui sont en relation avec les virus de la fièvre récurrente, notons les Leptospira dont le rôle chez les Chiroptères a été fort peu mis en évidence. En Afrique Centrale, il semble que la leptospirose est répandue par les chauves-souris très abondantes près des sources où s'abreuvent hommes et animaux; en Ethiopie, cette maladie est dénommée « bat-sickness » (Rizzotti 1939).

\section{D) Protozoaires intestinaux.}

Des Coccidies du genre Eimeria ont été détectées en Uganda: Eimeria dukei Lavier 1928 dans l'intestin de Tadarida pumila Cretzschmar (Stiles et Nolan 1930, p. 611).

Rien au Katanga.

\section{E) Helminthes.}

1. Trématodes.

a) Dicrocoelidés : Dictyonograptus pipistrelli Sandground $1937 a$ : dans la vésicule biliaire de Pipistrellus nanus Peters, à Ebombo (N.E. Katanga). - Eurytrema epomopis Sandground 1937 : dans le pancréas de Epomops dobsoni Bocage. - Platynosomum philippinorum congolensis Sandground 1937 : dans l'intestin de Myotis bocagei Thomas.

b) Strigéidés : Euparadistomum pipistrelli Sandground 1937: dans l'intestin de Pipistrellus nanus Peters.

c) Urotrématidés : Urotrema scabridum Braun: dans l'intestin de Myotis bocagei Thomas.

Nous avons extrait de l'intestin de plusieurs Pipistrellus nanus Peters (Mimbulu, Kilobelobe $=$ environs d'Elisabethville) des Trématodes encore indéterminés.

2. Nématodes.

a) Trichostrongylidés : Anoplostrongylus alatus Ortlepp 1932: dans l'intestin de Miniopterus schreibersi natalensis A. Smith.

b) Filariidés : Litomosa chiropterorum Ortlepp 1932: dans la cavité abdominale de Miniopterus schreibersi natalensis A. Smith. 
c) Ancylostomidés : Strongylacantha pretoriensis Ortlepp 1932: dans l'intestin de Rhino'ophus ciivosus zuluensis K. Andersen. - Une espèce, encore non identifiée, a été extraite du duodenum de Scotophilus nigrita Schreber, à Kipopo (Elisabethville).

d) Cucullanidés : Seuratum congolense Sandground $1937 b$ : dans le duodénum de Pipistrellus nanus Peters et de Myotis bocagei Thomas, à Kalanda (N. Katanga) et Kakonde (Kapolowe).

e) Physaloptéridés : Physaloptera bedfordi Ortlepp 1932: dans l'estomac de Rhinolophus clivosus zuluensis K. Andersen.

3. Cestodes.

Des œufs de Taenia sp. dans les se.les de Nycteris macrotis Dobson, au Keyberg (Elisabethville).

F) Acariens.

\section{Sarcoptidés :}

a) Notoedres (Metanoedres) miniopteri Fain 1959: dans la lèvre inférieure de Miniopterus inflatus Thomas et de Pipistrellus nanus Peters.

Notoedres (N.) mimetilli Fain 1959: dars la peau de l'aile de Mimetillus thomasi Hinton, au Katanga.

b) Nycteridocoptes eyndhoveni Fain 1959: dans les ailes de Rhinolophus clivosus zuluensis K. Andersen, Rhinolophus blasii empusa K. Andersen et Rhinolophus hildebrandti Peters (grottes de Lubudi).

Nycteridocoptes miniopteri Fain 1959 : dans les ailes de Miniopterus inflatus Thomas.

Nycteridocoptes rousetti Fain 1958 : dans la muqueuse buccale de Rousettus aegyptiacus E. Geoffroy.

c) Teinocoptes epomophori Rodhain 1923: chez diverses Roussettes épaulières.

2. Gastronyssidés (Fain 1959):

a) Opsonyssus brutsaerti Fain 1956: dans les fosses nasales et sur la cornée de diverses Roussettes.

b) Rodhainyssus yunkeri Fain 1956: dans les fosses nasales de Miniopterus inflatus Thomas.

\section{Listrophoridés :}

Parasites vivant des sécrétions de la peau, sans causer de préjudice à leur hôte : Myotis tricolor Temminck.

a) Alabidocarpus calcaratus Lawrence 1952.

b) Labidocarpus cristatus Lawrence 1953. 


\section{III. - ECTOPARASITES}

Les parasites externes des Chiroptères se rencontrent parmi les Acariens et les Insectes (Diptères, Hémiptères,' Siphonaptères). Nous donnons d'abord quelques précisions sur leur classification; nous grouperons ensuite les espèces suivant leurs hôtes en tenant compte seulement de notre matériel. Des remarques complémentaires préciseront certaines observations.

\section{A) Classification.}

ACARIENS.

1. Gamasoides : Macronyssidés (Dermanyssidés) et Spinturnicidés (Spinturnix, Ancystropus, Periglischrus).

2. Ixodoides : Ixodidés et Argasidés.

3. Trombidiformes : Trombicula, Psorergates.

INSECTES.

\section{Diptères :}

a) Pupipares:

Nyctéribiidés (Nycteribia, Penicillidia, Eucampsipoda, Trypselia).

Stréblidés (Nycteribosca, Raymondia).

b) Hématophages: Simuliidés, Culicidés (Anophelinae, Phlebotominae), Ceratopogonidés (Culicoides) et Calliphoridés.

2. Hémiptères : Cimicidés (Cacodmus, Afrocimex), Polyctenidés (Eoctenes) et Réduviidés (Emesinae).

3. Siphonaptères : Ischnopsyllidés (Chiropteropsylla, Lagaropsylla, Thaumapsylla).

B) Chiroptères et leurs parasites (voir cartes I et II).

\section{Pteropidés.}

a) Rousettus (Rousettus) aegyptiacus E. Geoffroy.

- Spinturnix lateralis Kolenati (1; 2: grotte Mwanga,

- Ancystropus leleupi Benoît 1959 (1).

- Ancystropus zelebori Kolenati (2 : grotte Mwanga).

- Eucampsipoda africanum Theodor (1).

- Nycteribosca africana Speiser (1; 2: grotte Mwanga).

- Nycteribosca alluaudi Falcoz (2: grotte Mwanga).

- Afrocimex leleupi Schouteden 1951 (1; 2: grotte Mwanga).

- Thaumapsylla breviceps orientalis Smit (2: grotte Mwanga).

b) Epomophorus crypturus Peters.

- Ancystropus zelebori Kolenati (Elisabethville). 


\section{Emballonuridés.}

a) Taphozous mauritianus E. Geoffroy.

- Trypselia blainvillei Leach (Elisabethville, Kapolowe).

b) Taphozous sudani Thomas (2: grotte Mwanga).

- Steatonyssus n. sp. (à l'étude).

- Eoctenes intermedius Speiser.

- Chiropteropsylla brockmani Rothschild.

\section{Molossidés.}

a) Tadarida (Chaerephon) spillmanni Monard.

- Steatonyssus (st.) crassisetosus Till et Owen 1964 (Kinke).

- Spinturnix walkerae Zumpt et Till (Kinke).

b) Tadarida (Mops) condylura niveiventer Cabrera et Ruxton.

- Dermanyssus sanguineus (Elisabethville).

- Chelanyssus aethiopicus (Keyberg: Elisabethville).

- Ichoronyssus sp. (Elisabethville).

- Spinturnix semilunaris M. et L. (Keyberg).

- Nycteribia $(N)$ schmidlii scotti Falcoz (Keyberg).

c) Tadarida (Tadarida) ansorgei Thomas.

- Lagaropsylla anciauxi Smit 1957 (Mwadingusha).

\section{Nycteridés.}

Nycteris m. macrotis Dobson.

- Trombicula nycteris Jadin (Keyberg).

- Eoctenes nycteridis Horvath (Keyberg, Route d'Elisabethville vers Kasenga, Shinkolobwe).

\section{Rhinolophidés.}

a) Rhinolophus h. hildebrandti Peters.

- Spinturnix semilunaris M. et L. (9 bis: grotte Kyasala).

- Nycteribia $(N)$ schmidlii scotti Falcoz (11).

- Nycteribia (Stylidia) hoogstraali Theodor (11).

- Nycteribosca africana Speiser (1).

- Simuliidés ( 9 bis : grotte Kyasala).

b) Rhinolophus landeri lobatus Peters.

- Spinturnix viduus Zumpt (9 bis: grotte Mulonga).

- Spinturnix walkerae Zumpt et Till ( 9 bis : grotte Mulonga).

- Penicillidia pachymela Speiser (9 bis: grotte Kyasala).

- Nycteribosca africana Speiser (1).

- Steatonyssus (st.) benoiti Till et Owen 1964 (2).

c) Hipposideros caffer centralis K. Andersen.

— Periglischrus moucheti Till 1958 (1).

- Psorergates hipposideros Fain 1959 (1).

- Nycteribia $(N)$ schmidlii scotti Falcoz (1).

- Penicillidia fulvida Bigot (1).

- Penicillidia pachymela Speiser $(1 ; 3 ; 9$ bis : grotte Kyasala). 
- Nycteribosca africana Speiser ( 9 bis : grotte de Kyantapo).

- Raymondia seminuda Jobling (1; 9 bis : grotte de Kyantapo).

- Raymondia setiloba Jobling (1; 9 bis : grotte Kyamakonde).

- Raymondia watersoni Jobling (1; 9 bis : grotte Kyamakonde).

- Calliphoridés (?) : myiase (3: grotte Etienne).

d) Cloeotis percivali australis Roberts.

- Eoctenes intermedius Speiser (4: grotte Dethioux).

\section{Vespertilionidés.}

a) Miniopterus inflatus Thomas.

- Hirstesia transvaalensis Zumpt (1).

- Spinturnix semilunaris M. et L. (1).

- Trombicula sp., dans les oreilles (1).

- Nycteribia $(N)$ schmidlii scotti Falcoz (1; Kasapa: Elisabethville).

- Penicillidia fulvida Bigot (11).

- Raymondia seminuda Jobling (1).

b) Miniopterus schreibersi natalensis A. Smith.

- Hirstesia transvaalensis Zumpt (11).

- Spinturnix semilunaris M. et L. (11).

- Nycteribia $(N)$ schmidlii scotti Falcoz (11).

c) Scotophilus n. nigrita Schreber.

- Steatonyssus (st.) brucei Lavoipierre (Jadotville).

- Spinturnix scotophili Zumpt et Till (Karavia : Elisabethville).

d) Myotis (Selysius) bocagei Thomas.

- Spinturnix walkerae Zumpt et Till (Kiswishi : Elisabethville ; Kapolowe).

e) Pipistrellus nanus Peters.

- Chelanyssus aethiopicus (Nakiluba: Elisabethville).

- Spinturnix walkerae (Elisabethville, Kapolowe).

- Steatonyssus (st.) brucei Lavoipierre (Kipopo: Elisabethville).

\section{f) Laephotis wintoni angolensis}

- Steatonyssus n. sp. : à Mumena (à l'étude).

- Spinturnix walkerae Zumpt et Till (Mumena).

- Rhipicephalus simus Koch (Mumena).

- Cacodmus villosus Stal (Mumena).

Nous avons encore de nombreux parasites indéterminés, et notamment pour les espèces de Chiroptères non reprises ci-dessus.

\section{C) Remarques complémentaires.}

\section{ACARIENS Ixodidés.}

Nous avons récolté Rhipicephalus simus Koch au pied d'un arbre abritant des Laephotis wintoni angolensis Monard, à Mumena (près d'Elisabethville), espèce qui parasite normalement des Carnivores et des Ongulés.

Les Tiques de ce genre jouent un rôle dans l'épidémiologie du « Tick Typhus » en Afrique. 
2. Diptères hÉMATOPhages.

a) Simuliidés.

Le 21 juillet 1962, nous avons capturé quelques Simulies (encore à l'étude) sur Rhinolophus $h$. hildebrandti Peters dans la grotte Kyasala (Lubudi), c'est-à-dire dans le même biotope où nous avons observé une filariose chez Miniopterus inflatus Thomas.

b) Culicidés.

- Anophelinae: La seule station connue pour Anopheles (Myzomyia) rodhaini Leleup et Lips 1950 est la grotte Kalomoni, à Kakontwe (Jadotville). Nous n'avons pas la preuve que cet Anophèle pique les Chiroptères, mais c'est fort possible.

- Phlebotominae: Plusieurs espèces cavernicoles parasitent les chauves-souris (Wanson 1942). Notre abondant matériel est dans les mains des spécialistes.

c) Calliphoridés.

Agents de myiases. Nous avons observé un seul cas sur le bras d'un Hipposideros caffer centralis K. Andersen, dans la grotte Etienne, à Mulungwishi.

\section{HÉMIPTÈres.}

a) Cimicidés : Afrocimex leleupi Schouteden 1951.

Nous avons vu de nombreux individus pompant le sang de Rousettus aegyptiacus E. Geoffroy, dans les grottes de Kakontwe et de Pempéré.

Ces Punaises ont une très large distribution géographique : elles existent dans la plupart des grottes du Katanga, réfugiées le plus souvent dans des fissures (parois et voûtes). Nous en avons trouvé gorgées de sang dans des grottes non fréquentées par les Roussettes, ce qui permet de penser qu'elles parasitent également des Microchiroptères (Rhinolophes et Minioptères).

\section{b) Reduviidés.}

Des Emésines se rencontrent dans plusieurs grottes du Katanga (Anciaux 1961), mais ne parasitent pas les Chiroptères.

4. Siphonaptères.

Lagaropsylla anciauxi Smit 1957 : à la fin de la saison sèche (septembre-octobre), la colonie de Tadarida ansorgei Thomas quitte Mwadingusha. Les Puces qui, gorgées de sang, ont abandonné leurs hôtes, s'attaquent alors à l'homme.

\section{IV. - VECTEURS CONNUS OU PROBABLES DE CES PARASITES}

\section{Infections à virus.}

Parmi les Acariens, des Ixodidés peuvent produire des toxines amenant des cas de paralysie, des Argasidés sont vecteurs de Spirochètes, des larves de Trombiculidés provoquent des fièvres et des typhus. 


\section{Parasites sanguins.}

a) Haemosporidies.

- Plasmodium : rôle possible des Anophèles cavernicoles.

- Hepatocystis epomophori Rodhain et Nycteria medusiformis Garnham et Heisch : transmetteurs inconnus.

- Polychromophilus :

- Garnham pense qu'il faut chercher les vecteurs dans le genre Culicoides (communication à M. Lips).

- Mer et Goldblum (1947) considèrent les Nycteribiidés (et surtout le genre Penicillidia) comme les principaux vecteurs; ils ont observé des sporozoïtes chez Penicillidia dufouri Westwood.

- Nous avons noté la présence de Nycteribia schmidlii scotti Falcoz chez toutes les espèces de Chiroptères parasités par Polychromophilus.

Pour les Haemosporidies, il ne faut pas écarter l'intervention des Phlébotomes.

b) Trypanosomes.

Nous pensons que les Hémiptères Cimicidés (et spécialement Afrocimex leleupi Schouteden) peuvent être considérés comme vecteurs de Trypanosomiases chez les Chiroptères du Katanga. Dans une punaise gorgée de sang, nous avons en effet observé des formes pré-trypanosomes (Crithidia et Leptomonas).

c) Microfilaires.

Diptères Simuliidés : transmetteurs possibles.

d) SPIRochètes.

Acariens du genre Ornithodoros: vecteurs probables.

\section{V. - LES HISTOPLASMES DANS LES GROTTES DU HAUT-KATANGA}

Avec la collaboration du Laboratoire Médical d'Elisabethville, nous avons isolé, le 22 décembre 1959, une souche d'Histoplasma capsulatum Darling à partir de souris blanches exposées dans la grotte Etienne à Mulungwishi. Nous avons publié deux notes sur cette question (Anciaux de Faveaux 1960, 1964 b).

Nous ne pensons pas que les Tiques puissent être des vecteurs de ces Champignons, contrairement à l'opinion de Coudert (1955). Certains auteurs (Emmons 1958; Baylet et al. 1958) attribuent un rôle épidémiologique aux Chiroptères. Lunn (1960) a étudié le cas d'un malade (atteint d'histoplasmose) ayant vécu dans la région de Kampala (Afrique Orientale) où beaucoup de chauves-souris habitent dans les arbres et les vieilles maisons ; des radiographies et des tests à l'histoplasmine n'ont pas donné de résultats positifs au point de vue relation avec les Chiroptères. Emmons et Greenhall (1962) ont isolé $H$. capsulatum Darling dans des biotopes (maisons, sous des racines adventives de kapokier, et dans une petite caverne à munitions abandonnée) fréquentés par les chauves-souris, à Trinidad. 
Au Katanga, 9 Rhinolophidés de la grotte Etienne (Mulungwishi) ont été radiographiés par le $\mathrm{D}^{r}$ Bovy ; un seul présentait une lésion pulmonaire. Sur la base d'un seul cas encore discutable, il nous paraît prématuré de tirer des conclusions sur l'influence des Chiroptères dans l'épidémiologie de l'Histoplasmose. Cependant, le D $\mathrm{D}^{r}$ J. Frederick Bell (Rocky Mountain Laboratory, Hamilton, Montana, U.S.A.) vient de découvrir un Myotis local infecté avec H. capsulatum Darling (coupes microscopiques).

\section{Résumé}

Etude des infections et maladies provoquées, directement ou indirectement, par les Chiroptères et leurs parasites.

Observations écologiques sur les Chiroptères du Haut-Katanga.

Endoparasites (bacilles, virus, parasites sanguins, Protozoaires intestinaux, Helminthes, Acariens).

Ectoparasites (Acariens ; Insectes : Diptères, Hémiptères, Siphonaptères).

Vecteurs connus ou probables de ces parasites.

Histoplasmes dans les grottes du Katanga : dissémination oossible par les Chiroptères.

Nous exprimons notre vive gratitude à nos collègues de la Société de Spéléologie du Katanga, ainsi qu'aux spécialistes qui ont eu l'obligeance de déterminer une partie de notre matériel : les Docteurs V. Aellen, H. Felten et R.-W. Hayman (Chiroptères), P. L. G. Benoît, W.-E. China, B. Jobling, H. E. Krampitz, M. Lips, F. G. A. M. Smit, W. Till, O. Theodor et A. Villiers (parasites). Nous remercions également M. Schweitzer, Directeur du Service topographique de l'Union Minière du Haut-Katanga, à Jadotville.

\section{Bibliographie des travaux cités}

Anciaux de Faveaux (M.-F.), 1950. - Cavernes (Guide de la Nature, Dinant ; 320 pp.).

—, 1956. - Aperçu préliminaire sur la Spéléologie du Katanga (Bull. Inf. Fédér. Spéléo. Belgique, t. 3, 9, pp. 3-4).

-, 1958 a. - Chiroptères des grottes du Haut-Katanga (Bull. Inst. Fr. Afr. Noire ; t. 20 ; série A ; 1 ; pp. 263-275).

—, 1958 b. - Les Chiroptères : utiles ou nuisibles? (Lovania, $\mathrm{n}^{\circ} 48$; pp. 45-51).

—, 1959-1960. - Notes biospéléologiques et parasitologiques sur des grottes du Katanga (Bull. Soc. Spéléo. Katanga, I, pp. 23-36 ; II, pp. 19-22).

-, 1960. - Considérations écologiques sur l'Histoplasmose au Katanga (Ann. Soc. Belge Méd. Trop. ; t. 40 ; pp. 298-303).

-, $1964 a$. - La faune des grottes du Haut-Katanga (III ${ }^{\circ}$ Congrès Intern. Spéléologie ; Vienne, 1961 ; t. III, section 2 ; pp. 11-21).

—, 1964 b. - L'Histoplasmose dans les grottes du Haut-Katanga (ibidem, pp. 7-10; 2 pl.). 
BeLL (J.-F.), 1963. - Communication personnelle (20/XI/63).

BENOIT (P.-L.-G.), 1959. - Un nouveau Spinturnicide sur Chauves-souris frugivores du Congo Belge (Rev. Zool. Bot. Afr.; t. 59 ; pp. 106-108).

Blanc (G.) et Bruneau (J.), 1957. - Présence chez une chauve-souris du Maroc d'un virus de Q. Fever (Bull. Soc. Path. Exot.; t. 50 ; pp. 653-656).

Boulger (L.-R.) et Porterfield (J.-S.) : Isolation of a virus from Nigerian fruit bat (Trans. Roy. Soc. Trop. Med. Hyg., t. 52, pp. 421-424).

- - et Hardy (J.), 1960. - Rabies in Nigeria (West Afr. Med. J., t. 9, 6, pp. 223-234).

Baylet (R.), Hubert et Quenum (C.), 1958. - Index histoplamique en A.O.F. (Bull. Soc. Path. Exot., t. 51, pp. 582-589.

Collier (W.-A.), 1955. - Die Epidemiologie der Parapoliomyelitisgruppe (Z. f. Tropenmedizin und Parasit., t. 6, pp. 243-251).

Constantine (D.-G.), 1962. - Rabies transmission by nonbite route. (Publ. Health Rep., Washington, t. 77, pp. 287-289).

Coudert (J.), 1955. - Guide pratique de Mycologie médicale (Masson, Paris, pp. 290-293).

De Meillon (B.), Davis (H.-S.) et Hardy (F.), 1961. - Plague in Southern Afrika (Vol. I : The Siphonaptera). Pretoria.

Emmons (C.-W.), 1958. - Association of bats with Histoplasmosis. (Publ. Health Rep., t. 73, pp. 590-595).

- et Greenhall (A.-M.), 1962. - Histoplasma capsulatum and house bats in Trinidad (Sabouraudia, II, p. 18-22).

FAIN (A.), 1959 a. - Le genre Teinocoptes Rodhain (Rev. Zool. Bot. Afr., t. 59, pp. 118136).

—, 1959 b. - Sur le genre Nycteridocoptes (Acarologia, t. 1, pp. 335-353).

-, 1959 c. - La famille Gastronyssidae (Bull. Inst. Roy. Sci. Nat. Belgique, t. 35, 12, 22 pp.).

-, 1959 d. - Le genre Notoedres (Rev. Zool. Bot. Afr., t. 60, pp. 131-167).

Garnham (P.-C.-C.) et Heisch (R.-B.), 1953. - On a new bloodparasite of insectivorous bats : Nycteria medusiformis. (Trans. Roy. Soc. Trop. Med. Hyg., t. 47, p. 357).

-, Heisch (R.-B.) et Furlong (M.), 1963. - Blood and tissu studies of Nycteria congolensis (Ibidem, t. 57, p. 232).

HeIsCH (R.-B.), 1953. - On a Spirochaete isolated from Ornithodoros graingeri (Parasitology, t. 43, pp. 131-135).

Krampitz (H.-E.) et Anciaux de Faveaux (M.-F.), 1960. - Ueber einige Haemosporidien aus Fledermäusen der Höhlen des Berglandes von Katanga (Z. f. Tropenmedizin und Parasit., t. 11, pp. 391-400).

LÉGer (M.) et BAURY (A.), 1923. - De l'emploi de la chauve-souris comme animal réactif de la Peste (Bull. Soc. Path. Exot., pp. 78-79).

Leleup (N.) et LiPS (M.), 1950-1951. - Anopheles rodhaini n. sp. au Katanga (Rev. Zool Bot. Afr., t. 43, pp. 303-308, t. 44, pp. 39-64).

Lips (M.) et Rodhain (J.), 1956. - Quelques Hématozoaires de petits Mammifères du HautKatanga (Ann. Parasit. hum. et comp., t. 31, pp. 485-487). 
LunN (H. F.), 1960. - A case of Histoplasmosis of bone in East Africa (J. Trop. Med. Hyg., t. 63 , pp. 175-180).

Mer (G. G.) et Goldblum (N.), 1947. - A Haemosporidian of bat (Nature, London, t. 159, p. 144).

Novel (J.), 1954. - Les spirochétoses des animaux sauvages (Mammalia, t. 18, pp. 279280).

OK1L.ePP (H. J.), 1932. - Some Helminths from S. Africa Chiroptera (18th Report of the Dir. vet. serv. and anim. Indust. Union S. Africa, t. 1, pp. 183-193).

Rizzotti (G.), 1939. - (Riv. Biol. Coloniale, Rome, t. 2, p. 241).

RodhaIn (J.), 1942. - Sur la spécificité morphologique et biologique de Trypanosoma pipistrelli (Acta Biol. Belgica, t. 2, p. 55).

—, 1951. - Trypanosoma leleupi n. sp., parasite de Hipposideros caffer au Katanga (Ann. Parasit. hum. et comp., t. 26, pp. 133-137).

Roshdy (M.-A.), 1962. - Comparative internal morphology of subgenera of Argas Ticks (Ixodoidea, Argasidae). 2. subgenus Chiropterargas: Argas boueti Roubaud et Colas-Belcour, 1933 (J. Parasit., t. 48, pp. 623-630).

SANDGRound (J.-H.), 1937 a. - Trématodes de chauves-souris du Congo Belge (Papers on Helminthology ; 30 Year Jubileum K.-J. Skrjabin, Moscou).

- 1937 b. - Three new parasites Nematodes from the Belgian Congo (Rev. Zool. Bot. Afr., t. 29, pp. 232-235).

Schoenaers (F.), 1950. - Présence au Katanga d'Ornithodoros megnini Dugès (Ann. Soc. Belge Méd. Trop., t. 30, pp. 1541-1543).

Schouteden (H.), 1951. - Un genre nouveau de Cimicides du Katanga (Rev. Zool. Bot. Afr., t. 44 , pp. 278-280).

SмIT (F.-G.-A.-M.), 1957. - Two new bat-fleas of the genus Lagaropsylla from the Belgian Congo (Rev. Zool. Bot. Afr., t. 56, pp. 263-268).

Stiles (C. W.) et Nolan (M. O.), 1930. - Key Catalogue of parasites reported for Chiroptera (Bats) with their possible public health importance (Nat. Inst. Health, Washington, Bull. $\mathrm{n}^{\circ}$ 155).

SuItor (E. C.) et WeIss (E.), 1961. - Isolation of a rickettsialike microorganism (Wolbachia persica n. sp.) from Argas persicus Oken (J. Infect. Dis., t. 108, pp. 95-106).

TIll (W.-M.), 1958. - Description de Periglischrus moucheti n. sp. (Rev. Suisse Zoologie, t. 65 , p. 252).

Till (W. M.) et Owen Evans (G.), 1964. - The genus Steatonyssus Kolenati (Bull. British Museum, Natural History, Zoology; vol. II, $\mathrm{n}^{\circ}$ 8, pp. 513-582).

VAN Riel (J.) et HiernauX-L'Hoest (D. et J.), 1951. - Description of a Plasmodium found in a Bat, Rousettus leachi (Parasitology, t. 41, p. 270).

Wanson (M.), 1942. - Sur la biologie des Phlébotomes congolais (Rev. Trav. Sci. Med. Congo Belge, t. 1, pp. 23-43). 